\title{
Abandono del tratamiento de la tuberculosis en Nicaragua: resultados de un estudio comparativo
}

\author{
Norma I. Soza Pineda, ${ }^{1}$ Susan M. Pereira ${ }^{1}$ y Maurício L. Barreto ${ }^{1}$
}

Forma de citar

Soza Pineda NI, Pereira SM, Barreto ML. Abandono del tratamiento de la tuberculosis en Nicaragua: resultados de un estudio comparativo. Rev Panam Salud Publica. 2005;17(4):271-8.

RESUMEN Objetivo. Identificar factores relacionados con el abandono (deserción) del tratamiento de la tuberculosis en centros de salud de los departamentos de Managua y Matagalpa, en Nicaragua. Métodos. Se diseñó un estudio de casos y testigos pareados por edad y por municipio de tratamiento. Se seleccionaron como casos 251 pacientes mayores de 15 años que abandonaron el tratamiento antituberculoso y como testigos pacientes que concluyeron la farmacoterapia (razón 1:1) durante el periodo de enero de 1998 a diciembre de 2001. Se obtuvieron datos de aspectos demográficos y socioeconómicos, hábitos de vida y características de la atención. Las variables se seleccionaron y agruparon utilizando un modelo teórico jerarquizado. Por medio de un análisis de regresión logística condicional, se estimó la razón de posibilidades (odds ratio, OR), con un intervalo de confianza de 95\% (IC95\%).

Resultados. Son factores de riesgo de abandono de la farmacoterapia antituberculosa: sexo masculino (OR: 2,51; IC 95\%: 1,63 a 3,94), residencia inestable o en la calle (OR: 3,08; IC95\%: 1,57 a 6,49), cambio de domicilio durante el tratamiento (OR: 4,22; IC95\%: 2,06 a 9,93), consumo de bebidas alcohólicas (OR: 5,25; IC95\%: 2,43 a 12,94), uso de drogas ilícitas (OR: 5,25; IC95\%: 2,43 a 12,94), dificultad de acceso a los servicios de salud (OR: 2,64; IC95\%: 1,39 a $5,29)$ y un concepto negativo de la atención recibida (OR: 5,33; IC95\%: 1,52 a 28,56).

Conclusión. Es indispensable establecer en los servicios de salud medidas que contribuyan a abatir el riesgo de abandono. Es importante recuperar la participación social del sector de la salud mediante acciones comunitarias.

Palabras clave Tuberculosis, quimioterapia, desistencia de paciente, factores de riesgo, Nicaragua.

La tuberculosis sigue siendo uno de los problemas de salud pública de mayor gravedad en el mundo. Se es-

\footnotetext{
Instituto de Salud Colectiva, Universidade Federal de Bahia. Toda correspondencia deberá dirigirse a: Norma Irene Soza Pineda, Instituto de Salud Colectiva, Universidade Federal de Bahia. Rua Padre Feijó 29, Canela, 40110-170, Salvador, Bahia, Brasil. Correo electrónico: normasoza@hotmail. com
}

tima que 8,4 millones de personas desarrollan la enfermedad cada año y entre 2 y 3 millones mueren en ese período. Existen ahora 20 millones de enfermos, de los cuales $95 \%$ viven en países en desarrollo, donde ocurren $98 \%$ de las muertes por esta causa $(1,2)$.

El abandono del tratamiento se considera uno de los principales obstáculos para el control de la tuberculosis, porque disminuye la eficacia de los programas nacionales al incrementar el riesgo de transmisión del bacilo y aumentar la probabilidad de resistencia bacteriana a los antibióticos, la cual predispone a una mayor frecuencia de recidivas y fracasos (3-5).

La Organización Mundial de la Salud (OMS) (6) considera como abandono el incumplimiento por el pa- 
ciente del régimen farmacoterapéutico por un período mayor de dos meses. Algunos autores lo definen como la inasistencia continua del paciente a la unidad de salud por un período mayor de 29 días (4, 7-10); otros, como la inasistencia a los servicios de salud por tiempo indefinido, independientemente del tiempo que dure el tratamiento $(5,11,12)$. La normativa nacional de Nicaragua define como abandono la inasistencia por 30 días consecutivos a los servicios de salud (13). Esta fue la definición utilizada en el presente estudio.

Desde 1984, la estrategia de tratamiento supervisado se instauró en Nicaragua como tratamiento padrón en el sistema nacional de salud. Durante el decenio de 1980 se han registrado cerca de 3500 casos de tuberculosis por año. En los últimos 10 años han disminuido, de tal modo que ahora se notifican aproximadamente 2500 casos nuevos cada año. Entre 1990 y 2000, la incidencia osciló entre 66 y 47 casos por 100000 habitantes. En poco más de $60 \%$ de los casos se trata de pacientes bacilíferos, el grupo de edad más afectado es el de 25 a 34 años y el sexo con mayor registro de casos es el masculino (1, 14-16).

La evaluación de cohortes de pacientes revela que durante ese mismo decenio la tasa de curación aumentó en Nicaragua de $74,0 \%$ a $82,0 \%$, mientras que la mortalidad se redujo de 6,7 a 2,0 por 100000 habitantes y la deserción disminuyó progresivamente de $15,0 \%$ a $9,0 \%$. A pesar de esos avances, la tuberculosis se ratifica como la principal causa de muerte entre las enfermedades infecciosas en el grupo mayor de 35 años (1, 14-16).

En vista de que el abandono de la farmacoterapia antituberculosa sigue significando un fuerte obstáculo para incrementar la tasa de curación; que se han realizado pocos estudios sobre este fenómeno según se manifiesta en Nicaragua, y que factores relacionados con los pacientes y con la organización de los servicios de salud parecen contribuir al problema, el presente estudio se fijó el objetivo de identificar factores demográficos y socioeco- nómicos, hábitos de vida de los pacientes y características de los servicios de salud, relacionados con el abandono del tratamiento de la tuberculosis en Nicaragua.

\section{MATERIALES Y MÉTODOS}

Se realizó un estudio de casos y testigos $(17,18)$, pareados por edad y por municipio de tratamiento, para evaluar el abandono de la farmacoterapia antituberculosa entre pacientes inscritos en el Programa de Control de la Tuberculosis (PCT) de Nicaragua, durante el período de enero de 1998 a diciembre de 2001, en los centros de salud de los Sistemas Locales de Atención Integral a la Salud (SISAIS) de los departamentos de Managua y Matagalpa.

El departamento de Managua, que aloja a la ciudad de Managua, capital del país, se localiza en la zona del Pacífico, con una población estimada para el año 2000 de 1262660 habitantes, lo que representa $25 \%$ de la población nacional. El departamento se divide en 9 municipios, con 21 unidades de atención primaria de salud. En él se registran cada año unos 800 casos nuevos de tuberculosis (lo que representa $30 \%$ de la cifra nacional). Por otro lado, el departamento de Matagalpa está situado en la zona central de Nicaragua, con una población estimada de 539325 habitantes, lo que corresponde a $11 \%$ de la población del país. A diferencia del departamento de Managua, el de Matagalpa presenta un relieve geográfico montañoso, lo que determina cierto grado de inaccesibilidad geográfica. Matagalpa está dividida en 15 municipios, con sus respectivos centros de salud $(1,14,15)$.

La farmacoterapia de la tuberculosis tiene una duración de ocho meses. Se administra bajo supervisón directa durante la primera fase y en la segunda es autoadministrada, en todas las formas clínicas. En los casos de recaída, fracaso o abandono, se administra un esquema de repetición de la farmacoterapia (retratamiento), el cual implica la hospitalización del paciente durante la primera fase (3 meses) y su- pervisión directa durante la segunda (5 meses) (13).

Según el Manual del Programa de Control de Tuberculosis, de Nicaragua, caso de tuberculosis es todo individuo con sospecha de la enfermedad cuyo examen microscópico de esputo demuestre la presencia de bacilos tuberculosos; también es caso de tuberculosis todo individuo con sospecha clínica o radiológica de la enfermedad, aun sin confirmación bacteriológica.

En nuestro estudio, el diagnóstico fue establecido previamente por el personal de salud del programa. Se consideró como casos a los individuos con registro de tuberculosis de cualquier forma clínica, con edad de 15 años o más, cuyo egreso del programa consistió en abandono. Los testigos (controles) fueron individuos cuyo resultado de tratamiento fue el alta por curación comprobada por baciloscopia o la curación no comprobada con tratamiento terminado, con edad similar a la de los casos ( \pm 3 años) y atendidos en los mismos servicios. Se designó un testigo por cada caso, siguiendo el orden de registro en el programa hasta encontrar el primer individuo que cumpliera los criterios. Se excluyó a cualquier posible caso o testigo cuyo resultado de tratamiento difiriera del criterio definido para cada grupo del estudio. La muestra total se calculó con la fórmula de Schlesselman (17), conforme a los parámetros siguientes: potencia de $80 \%$, intervalo de confianza de $95 \%$, riesgo estimado de 2,0, y frecuencia de exposición en los testigos de $10 \%$. La muestra del estudio estuvo integrada por 278 casos con sus respectivos testigos, para un valor $n$ total de 668 individuos, incluidos 112 correspondientes a las pérdidas.

Se consideró como variable dependiente el resultado del tratamiento, que se clasificó en: 1) abandono —toda inasistencia a tratamiento mayor de 30 días-, o 2) cura-la conclusión del tratamiento, con comprobación o no por baciloscopia. Las variables independientes se clasificaron en tres grupos en función de: 1) características demográficas y socioeconómicas, 2) hábitos de vida, y 3) antecedentes 
clínicos y características de la atención en los servicios de salud.

Entre las variables demográficas y socioeconómicas, el hacinamiento se cuantificó según el número de personas por cuarto o dormitorio: más de dos personas $=1$; dos personas $\mathrm{o}$ menos por cuarto $=0$. En cuanto a las variables relacionadas con hábitos de vida del paciente, el alcoholismo se evaluó según el cuestionario QUEST: dos o más preguntas positivas $=1$; ninguna o solamente una positiva $=0$. Se consideró fumador aquel individuo que refirió tener 6 meses o más de fumar. Entre las características de la atención en los servicios de salud se incluyeron como dificultades para el acceso: problemas económicos, horario de trabajo, distancia de traslado a la unidad de salud, falta de ayuda para encargar a los hijos o debilitamiento físico. Como dificultades para recibir el tratamiento en la unidad de salud se consideraron: unidad de salud que no brinda atención especializada, personal del programa no presente en la unidad, restricciones para la obtención del medicamento y largo tiempo de espera.

Para obtener la información se visitaron las unidades de salud y se procedió a revisar los expedientes médicos de los pacientes y a llenar una ficha de información. Posteriormente, se realizaron visitas domiciliarias para entrevistar a los pacientes. Los entrevistadores fueron trabajadores de salud, diferentes del personal del programa y adscritos a las unidades de salud del estudio.

De las 19 unidades de salud de Managua, debieron excluirse cinco centros por las razones siguientes: falta de recursos humanos para obtener la información (2), ningún caso de abandono registrado en ese período (2) y falta de servicio especializado para pacientes tuberculosos (1). En Matagalpa, de 15 centros de salud, solo dos no registraron casos de abandono durante el período de estudio. La población de estudio incluyó a todos los pacientes que abandonaron el tratamiento - un total de 367 casos-, con su respectivo testigo curado. De los casos de abandono,
265 se registraron en Managua $(72,2 \%)$ y 102 en Matagalpa $(27,8 \%)$.

Los datos se registraron en el programa Epi Info (versión 6.04) ${ }^{2}$ y se procedió a realizar un análisis de coherencia. Como primer paso se realizó un análisis descriptivo unifactorial (de variable única) y luego bifactorial (de dos variables) entre los factores de riesgo y el abandono del tratamiento. Se calculó la razón de posibilidades (odds ratio, OR) y el intervalo de confianza de 95\% respectivo (IC95\%). Todas las variables que mostraron asociación estadísticamente significativa en esta etapa fueron incluidas en el análisis multifactorial (de variables múltiples). En todas las etapas del análisis se utilizó el programa Stata (versión 7.0). ${ }^{3}$

Para el análisis multifactorial se utilizó la estrategia de análisis jerarquizado en la selección de variables, la cual se basa en un modelo teórico que describe la relación jerárquica o paralela entre varios grupos de factores de riesgo referentes a una enfermedad o problema de salud determinado. El criterio para la inclusión de esos factores dentro del modelo requiere no solo una relación estadística con el problema, sino también la consideración de los determinantes sociales, económicos y biológicos. Estos factores pueden no ser causa directa del problema, de modo que se clasifican como determinantes distales, y los condicionantes directos, como determinantes proximales.

A diferencia de otros modelos, el análisis jerarquizado se propone adecuar el ajuste de determinantes considerados distales por los proximales, principalmente los relacionados con las condiciones de vida. El modelo también presupone que existen diferencias entre los factores, de modo que considera el efecto directo o indirecto de cada uno de ellos sobre el problema en estudio $(19,20)$.

De esta forma, siguiendo un modelo jerarquizado, las variables se agrupa-

2 Epi Info versión 6,04d (enero de 2001), proporcionado por los Centros para el Control y la Prevención de Enfermedades, de Estados Unidos.

3 Stata Corporation. Statistics Data Analysis. Copyright 1984-2001. ron en tres bloques (figura 1) según se relacionaran con:

- bloque 1: características socioeconómicas y demográficas,

- bloque 2: hábitos de vida, y

- bloque 3: características de la atención clínica.

En el análisis se utilizó el modelo de regresión logística condicional para estimar el valor de la razón de posibilidades (OR) y sus respectivos intervalos de confianza de 95\%. La relación entre el abandono y las diferentes variables se consideró estadísticamente significativa cuando el intervalo no incluía el valor nulo (es decir, cuando OR = 1) (17).

Las variables del primer bloque que en la primera etapa presentaron una asociación positiva y estadísticamente significativa con el abandono continuaron formando parte del modelo en las etapas siguientes, excepto, solamente, las no asociadas de los tres bloques respectivos. El modelo final estuvo compuesto por las variables seleccionadas en cada etapa del análisis, con sus respectivos niveles de significación estadística observados inicialmente.

\section{RESULTADOS}

Se entrevistó a 313 casos y 336 testigos. Las pérdidas se debieron a recaídas de un mismo paciente, extravío o ausencia de registros clínicos, imposibilidad de localizar a algunos pacientes en la dirección conocida y la eventualidad de que un paciente se encontrara hospitalizado durante la etapa de entrevistas. Concluida la revisión de los cuestionarios, se excluyeron 62 casos y 85 testigos porque brindaban poca información sobre las variables principales del estudio. De esta manera, participaron en el análisis final 502 individuos (251 pares de casos y testigos), lo que constituye $90,2 \%$ de la muestra estimada. Por cada individuo incluido se llenó la ficha de información del expediente clínico. Fue posible entrevistar en su casa solamente a 490 pacientes, debido a cambio de domicilio o a fallecimiento. 
FIGURA 1. Modelo jerárquico de la relación entre los factores de riesgo de abandono del tratamiento antituberculoso

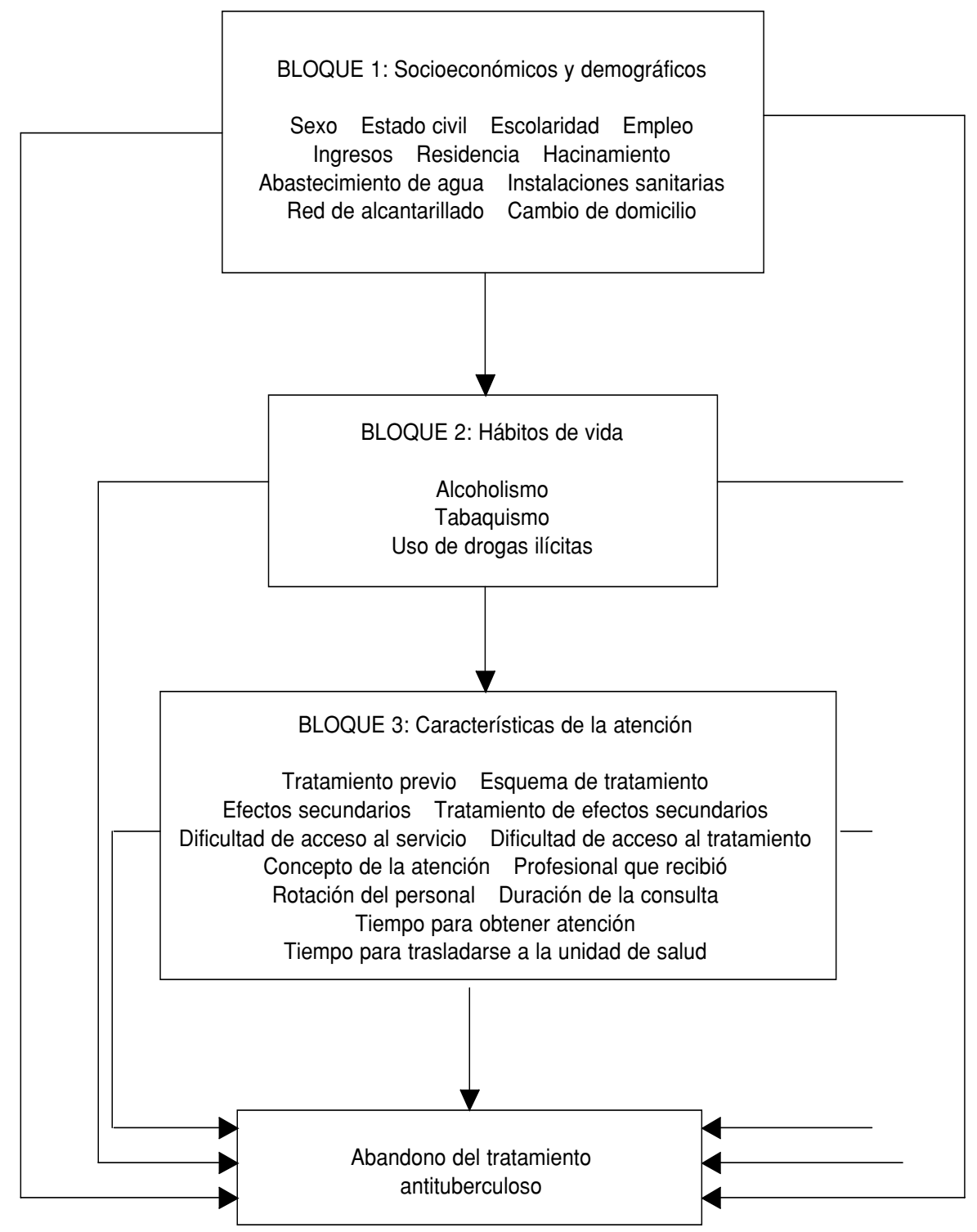

En el cuadro 1 se aprecia una distribución similar entre la población de estudio según las variables de pareamiento. En relación con las características demográficas y socioeconómicas, se observó que los varones predominaron tanto en el grupo de casos como en el de los testigos, una asociación que fue positiva por una OR de 2,51 (IC95\%: 1,63-3,94) y estadísticamente significativa, con el abandono del tratamiento. Entre los que abandonaron y los que se curaron no hubo diferencias en cuanto al estado civil; un poco más de la mitad de todos los pacientes refirieron no tener pareja estable. El analfabetismo o la baja escolaridad predominaron entre los pacientes que desertaron. Más de la mitad de los abandonos correspondieron a individuos desempleados y, de los que trabajaban, $80 \%$ recibían menos de un salario mínimo. Quienes vivían en la calle o no tenían domicilio estable presentaron un riesgo tres veces mayor de desistir de la farmacoterapia (OR: 3.08;
IC95\%: 1,57-6,49), una asociación que resultó estadísticamente significativa.

Las condiciones de hacinamiento, la carencia de servicios básicos dentro de la vivienda y la falta del servicio de alcantarillado no se vieron relacionadas con el abandono de la farmacoterapia. Quienes cambiaron de residencia durante el tratamiento presentaron un riesgo cuatro veces mayor de desertar que los que permanecieron en el mismo domicilio, una asociación que fue positiva (OR: 4,22; IC95\%: 2,06$9,93)$ y estadísticamente significativa.

En cuanto a los hábitos de vida (cuadro 2), se observó que el número de individuos que fumaban (OR: 4,83; IC95\%: 2,06-9,93), consumían bebidas alcohólicas (OR: 5,25; IC95\%: 2,069,93 ) o usaban drogas ilícitas (OR: 5,25; IC95\%: 2,06-9,93) fue proporcionalmente mayor entre los casos que entre los testigos; el riesgo de abandonar el tratamiento fue casi cinco veces mayor cuando el paciente utilizaba alguna de esas sustancias.

En cuanto a la atención clínica, se observó que la forma clínica predominante fue la pulmonar, tanto en los casos (93\%) como en los testigos (93\%), una asociación que no fue estadísticamente significativa. El riesgo de abandonar la farmacoterapia aumentó al doble cuando había ya antecedente de tratamiento antituberculoso (OR: 2,14; IC95\%: 1,25-3,78). Los que siguieron un esquema terapéutico distinto del régimen abreviado presentaron una asociación positiva (OR: 1,77; IC95\%: 1,12-2,85), estadísticamente significativa, con el abandono. Solamente la asociación entre la presencia de efectos secundarios y el abandono del tratamiento resultó negativa, con una diferencia estadísticamente significativa (OR: 0,51; IC95\%: 0,25-0,99). La medicación contra efectos secundarios no mostró relación estadísticamente significativa. El riesgo de deserción fue 2,6 veces mayor cuando el paciente enfrentaba dificultades para acceder a los servicios de salud (OR: 2,64; IC95\%: 1,39-5,29). Otra condición importante fue el concepto negativo del paciente acerca de la calidad de la atención, en cuyo caso el riesgo de deserción se in- 
CUADRO 1. Descripción de la población de estudio según las variables de pareamiento. SILAIS, ${ }^{a}$ Managua-Matagalpa, 1998-2001

\begin{tabular}{|c|c|c|c|c|c|}
\hline \multirow[b]{2}{*}{ Variable } & \multicolumn{2}{|c|}{ Casos } & \multicolumn{2}{|c|}{ Testigos } & \multirow[b]{2}{*}{$P$} \\
\hline & $n$ & $\%$ & $n$ & $\%$ & \\
\hline \multicolumn{6}{|l|}{ Edad } \\
\hline 15-24 & 62 & 25 & 62 & 25 & 0,99 \\
\hline $25-34$ & 62 & 25 & 62 & 25 & \\
\hline $35-44$ & 63 & 23 & 63 & 25 & \\
\hline$\geq 45$ & 64 & 25 & 64 & 25 & \\
\hline \multicolumn{6}{|c|}{ Tipo de municipio } \\
\hline Urbano & 165 & 66 & 165 & 66 & 1,00 \\
\hline Rural & 86 & 34 & 86 & 34 & \\
\hline
\end{tabular}

Fuente: Ministerio de Salud de Nicaragua. Libro de Registro del Programa de Control de la Tuberculosis, 2001-2002.

a SILAIS, Sistemas Locales de Atención Integral a la Salud (Nicaragua).

CUADRO 2. Razón de posibilidades (OR) no ajustada de la asociación entre el abandono

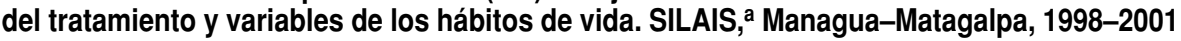

\begin{tabular}{|c|c|c|c|c|c|c|}
\hline \multirow[b]{2}{*}{ Variable } & \multicolumn{2}{|c|}{ Casos } & \multicolumn{2}{|c|}{ Testigos } & \multirow[b]{2}{*}{$\mathrm{OR}^{\mathrm{b}}$} & \multirow[b]{2}{*}{ IC95\% ${ }^{c}$} \\
\hline & $n$ & $\%$ & $n$ & $\%$ & & \\
\hline \multicolumn{7}{|l|}{ Hábito de fumar } \\
\hline No & 94 & 53,70 & 140 & 80,00 & & \\
\hline $\mathrm{Si}$ & 81 & 46,30 & 35 & 20,00 & 4,83 & $2,06-9,93$ \\
\hline Total & 175 & & 175 & & & \\
\hline \multicolumn{7}{|c|}{ Consumo de bebidas alcohólicas } \\
\hline No & 126 & 72,40 & 160 & 92,00 & & \\
\hline $\mathrm{Si}$ & 48 & 27,60 & 14 & 8,00 & 5,25 & $2,06-9,93$ \\
\hline Total & 174 & & 174 & & & \\
\hline \multicolumn{7}{|l|}{ Uso de drogas ilícitas } \\
\hline No & 138 & 75,00 & 172 & 93,40 & & \\
\hline $\mathrm{Si}$ & 46 & 25,00 & 12 & 6,60 & 5,25 & $2,43-12,94$ \\
\hline Total & 184 & & 184 & & & \\
\hline
\end{tabular}

Fuente: Entrevistas domiciliarias con los pacientes; población de SILAIS.

a SILAIS, Sistemas Locales de Atención Integral a la Salud (Nicaragua).

${ }^{b}$ Razones de disparidad (odds ratios, OR) y respectivos

${ }^{c}$ Intervalos de confianza obtenidos mediante análisis de regresión logística condicional.

crementó en cinco tantos (OR: 5,33; IC95\%: 1,52-28,56). Otras características de los servicios de salud, como dificultades de acceso al tratamiento dentro de las unidades, perfil del profesional de salud que recibió el ingreso, rotación del personal del programa y duración de la consulta, no fueron estadísticamente significativos. Casi $80 \%$ de los desertores y de los curados buscaron atención médica tardíamente, a pesar de requerir menos de una hora para trasladarse a la unidad de salud más próxima a su domicilio. Ninguna de estas dos asociaciones fue estadísticamente significativa.
El modelo final estuvo compuesto de las variables seleccionadas en cada etapa del análisis, con sus respectivos niveles de significación estadística observados inicialmente (cuadro 3). De esta manera, se identificaron como factores de riesgo de abandono del tratamiento antituberculoso: pertenecer al sexo masculino, carecer de residencia fija o vivir en la calle, cambiar de domicilio durante el tratamiento, consumir bebidas alcohólicas, usar drogas ilícitas, enfrentar dificultades de acceso a los servicios de salud y tener una impresión negativa de la atención clínica recibida.

\section{DISCUSIÓN}

Los resultados de este estudio indican que factores ligados al sexo, a las condiciones socioeconómicas y a los hábitos de vida, así como algunas características de los servicios de salud, contribuyen al abandono de la farmacoterapia antituberculosa.

Las diferencias biológicas entre hombres y mujeres se convierten en un problema cuando se consideran factores ligados al sexo, características socioeconómicas y culturales. El riesgo de exposición a fuentes de infección de la tuberculosis es mayor si se tienen en cuenta esos factores y si se relacionan con diferencias en los hábitos de vida, como alcoholismo, tabaquismo y drogadicción.

En este estudio, los pacientes del sexo masculino presentaron un riesgo casi dos veces y medio mayor de desistir de la farmacoterapia que las mujeres. Ya otros estudios descriptivos han mostrado mayor propensión de los varones a abandonar el tratamiento $(4,8,9,12$, 21, 22). La asociación estimada entre la población indígena de Rondônia, Brasil, fue menor que la de nuestros resultados (OR: 1,9) (23). En Chile (11) se estimó un riesgo relativo superior (RR: 3,1) al de nuestros resultados. Otros estudios no demostraron asociación entre sexo masculino y abandono del tratamiento (5, $12,24)$. Es probable que esas diferencias se deban a los contextos distintos de esos estudios y a la utilización de diseños metodológicos diferentes.

La falta de un domicilio fijo o el vivir en la calle se relacionan con un riesgo tres veces mayor de abandonar la farmacoterapia antituberculosa que el relacionado con la residencia estable. En busca de nuevos lugares donde vivir, las familias muy pobres y numerosas se mudan a zonas no urbanizadas que se configuran como asentamientos urbanos temporales. Esa movilidad ocasiona la inasistencia del paciente a las unidades de salud. Por su parte, los servicios de salud pierden el control de la localización de los inasistentes, lo que contribuye al abandono del tratamiento. Morar en la calle agrava aun más esas dificultades, al tornar impo- 
CUADRO 3. Características sociodemográficas, hábitos de vida y características de la atención clínica, como factores de riesgo de abandono del tratamiento antituberculoso. SILAIS, ${ }^{a}$ Managua-Matagalpa, 1998-2001

\begin{tabular}{|c|c|c|c|}
\hline Variable & $\begin{array}{c}\text { BLOQUE 1: } \\
\text { Características } \\
\text { socioeconómicas } \\
\text { y demográficas } \\
\text { OR ajustada (IC95\%) }\end{array}$ & 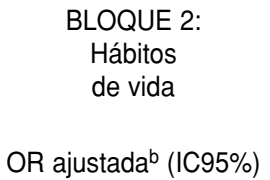 & $\begin{array}{c}\text { BLOQUE 3: } \\
\text { Características } \\
\text { de la } \\
\text { atención clínica } \\
\text { OR ajustada }{ }^{\mathrm{C}}(\mathrm{IC} 95 \%)\end{array}$ \\
\hline Sexo masculino & $3,50(1,96-6,25)$ & - & - \\
\hline $\begin{array}{l}\text { Residencia inestable o en } \\
\text { la calle }\end{array}$ & $3,00(1,44-6,23)$ & - & - \\
\hline Cambios de domicilio & $3,48(1,57-7,69)$ & - & - \\
\hline $\begin{array}{l}\text { Consumo de bebidas } \\
\text { alcohólicas }\end{array}$ & - & $3,88(1,55-9,68)$ & - \\
\hline Uso de drogas ilícitas & - & $3,89(1,57-9,61)$ & - \\
\hline $\begin{array}{l}\text { Dificultades de acceso } \\
\text { al servicio }\end{array}$ & - & - & $3,32(1,10-9,96)$ \\
\hline $\begin{array}{l}\text { Concepto negativo de la } \\
\text { atención clínica }\end{array}$ & - & - & $11,91(2,11-67,04)$ \\
\hline
\end{tabular}

Fuente: Entrevistas domiciliarias con los pacientes; población de SILAIS.

a SILAIS, Sistemas Locales de Atención Integral a la Salud (Nicaragua).

${ }^{b}$ Razón de disparidad (OR) ajustada por las variables: sexo, residencia y cambios de domicilio.

${ }^{\mathrm{C}}$ Razón de disparidad (OR) ajustada por las variables: sexo, residencia, cambios de domicilio, consumo de bebidas alcohólicas y uso de drogas ilícitas.

sible la localización de los enfermos. Otros autores (25), al investigar los factores relacionados con el incumplimiento de la DOTS, concluyeron que residir en la calle conlleva un riesgo de abandonar el tratamiento 3,2 veces mayor que el relacionado con tener una residencia estable. En otro estudio de esos mismos autores, en el que se evaluó el encarcelamiento como medida para disminuir el incumplimiento del régimen terapéutico, 95\% de los pacientes que estaban presos referían el antecedente de haber vivido en la calle (21).

El cambio de domicilio durante el tratamiento fue otro factor que mostró relación con el abandono. Esa condición, aunque ligada a la residencia provisional, responde más a los movimientos migratorios de la población en busca de fuentes de trabajo. Se estima que en la ciudad de Managua se concentran $40 \%$ de las migraciones internas. En el país, dos tercios de los migrantes viven en ciudades y un tercio en regiones rurales (1) .

En general, el consumo de bebidas alcohólicas se corresponde con factores culturales y sociales determinados. A menudo se asocia con el uso de tabaco y drogas ilícitas; en las etapas avanzadas de la enfermedad predis- pone al desempleo y la morada en la calle. Nuestro estudio demostró que el riesgo de deserción fue cinco veces mayor entre los consumidores de bebidas alcohólicas, una cifra similar a estimaciones anteriores referentes a Nicaragua (26) y superior a las obtenidas en Brasil (27) y en Estados Unidos (25). En este último país, el alcoholismo mostró relación relevante con el abandono de la estrategia DOTS. Nuestros resultados fueron compatibles con los de otros estudios descriptivos $(4,11)$ que demostraron una alta proporción de pacientes alcohólicos entre los que abandonaron el tratamiento. Este fue también un factor predictivo de abandono en São Paulo (12) y Río de Janeiro, Brasil (10). Nuestros resultados difirieron de los de un estudio realizado en Madrid, España (5), en el que no se identificó esa asociación entre pacientes infectados por el VIH. Según la literatura consultada, el consumo de bebidas alcohólicas es uno de los factores de riesgo más importantes para el abandono del tratamiento. Nuestros resultados corroboran esta asociación.

En comparación con el consumo de bebidas alcohólicas, los pacientes de Nicaragua refieren con menor frecuencia el consumo de drogas ilícitas. No obstante, es un problema social de gravedad creciente. En nuestro estudio quienes usaban drogas ilícitas mostraron un riesgo cinco veces mayor de abandonar el tratamiento que los que no las consumían. Es importante destacar que esa asociación fue igual a la observada con el consumo de bebidas alcohólicas. Condiciones de pobreza, desempleo, morada en la calle y alcoholismo pueden interferir en esa situación. En Madrid, España (5), el uso de drogas ilícitas, principalmente del tipo inyectable, fue identificado como único factor predictivo de abandono del tratamiento entre pacientes positivos al $\mathrm{VIH}$, con un riesgo doblemente mayor que el identificado en este estudio.

Las dificultades de acceso a los servicios de salud fueron expresadas por los pacientes en términos de problemas de transporte, económicos y del propio estado de salud. Nuestros resultados mostraron que el riesgo de deserción fue aproximadamente tres veces mayor entre aquellos con alguna dificultad para acceder a los servicios de salud. En México (8), se demostró una fuerte asociación entre abandono y residencia en el medio rural, la cual se traduce en cierto modo en dificultades de acceso geográfico. Este aspecto es pertinente a los servicios de salud de Nicaragua, puesto que el bajo nivel socioeconómico que caracteriza a la población en general redunda en dificultad de acceso a los servicios. Una proporción de la población rural reside en zonas con dificultades geográficas para el transporte, lo que también disminuye el acceso a las unidades de salud.

En nuestra experiencia, la aparición de efectos secundarios a los medicamentos incrementa la demanda de una atención médica más frecuente, de manera que el personal a cargo pasa a tener mayor control de estos casos. En contraposición con los resultados de otros estudios $(11,28)$, los pacientes que en nuestra muestra presentaron algún efecto secundario a los medicamentos no abandonaron la farmacoterapia.

Un concepto negativo sobre la calidad de la atención puede reducir el interés del paciente por cumplir con el tratamiento, así como contribuir a su 
decisión de no acudir a las mismas unidades cuando ocurren crisis ulteriores. En nuestro país la mayor parte de la población procura los servicios públicos de salud y el programa de control de la tuberculosis es administrado exclusivamente por esos servicios; por consiguiente, su opinión sobre la calidad de la atención tiene repercusiones importantes en el control del abandono. En nuestro estudio, este aspecto fue relevante, y estuvo representado por un riesgo cinco veces mayor de desistir de la farmacoterapia entre los pacientes que refirieron tener un concepto negativo de la atención recibida. En Río de Janeiro, Brasil (10), la declaración del paciente de que no retornaría al servicio actual y que buscaría otro mostró una fuerte relación con el abandono. En México (28), un estudio cualitativo mostró asociación entre la falta de comunicación médico-paciente $\mathrm{y}$ el incumplimiento del tratamiento. En nuestra muestra, únicamente 12,4\% de los pacientes entrevistados refirieron tener un concepto negativo de la atención clínica recibida. Sin embargo, consideramos que la relación entre el personal de salud del PCT y los pacientes necesita explorarse más a fondo, para identificar algunas situaciones que puedan todavía incidir en el abandono del tratamiento.

Entre las limitaciones de este estudio se identificaron posibles sesgos de selección referentes a la posibilidad de que hayan ocurrido casos más graves que culminaran en muerte sin poder ser registrados por los servicios de salud, o bien, a las dificultades propias de la selección de los testigos; además, en un principio no fue posible estable- cer contacto con todos los individuos seleccionados. No obstante, consideramos que esas posibilidades se llevaron al mínimo mediante la inclusión del mayor número de casos posible, además de que la mortalidad por esta causa ha disminuido progresivamente en Nicaragua. En la medida en que la escasa información sobre infección por VIH en esos pacientes corresponde a la baja tasa de prevalencia registrada en el país, otras variables pueden no haber alcanzado significación estadística, por el menor número de observaciones registradas. Para disminuir sesgos por datos incompletos en los expedientes clínicos, excluimos de la muestra final a aquellos individuos con mayor pérdida de información.

En vista de que en este estudio tanto casos como testigos se originaron en una misma base poblacional, de que el pareamiento por edad llevó al mínimo posibles efectos de confusión por esa variable y de que logró establecerse la comparabilidad de las variables entre casos y testigos, consideramos que los resultados del presente trabajo pueden hacerse extensivos a la población general de Nicaragua.

A partir del modelo teórico utilizado en este estudio, se concluye que son factores de riesgo para abandonar la farmacoterapia antituberculosa en Nicaragua: sexo masculino, residencia inestable o en la calle, cambios de domicilio durante el tratamiento, consumo de bebidas alcohólicas, uso de drogas ilícitas, dificultades de acceso a los servicios de salud y un concepto negativo de la atención recibida. Resulta evidente la necesidad de lograr una pronta identificación de estas ca- racterísticas a fin de establecer medidas que disminuyan el riesgo de que se abandone el tratamiento. En particular, se considera importante recuperar la participación social mediante la capacitación y el compromiso de los agentes comunitarios, grupos religiosos y organizaciones civiles que puedan apoyar las actividades pertinentes de salud pública en la comunidad.

\section{AGRADECIMIENTO}

Este estudio se hizo posible gracias al apoyo financiero de la Organización Panamericana de la Salud (OPS), por conducto del Programa de Subvenciones para la Investigación. Agradecemos también el apoyo y la participación de las autoridades del Ministerio de Salud de Nicaragua relacionadas con el estudio, principalmente a Alejandro Tardencilla, Coordinador Nacional del PCT, y a Joaquín Ufión Caldera, por sus valiosas críticas. Asimismo, a Marcia Cordero, por su valiosa colaboración en la coordinación del proceso de recopilación de la información en Matagalpa. Vaya un agradecimiento especial a los trabajadores y trabajadoras de las unidades de salud que recopilaron esa información. El presente trabajo forma parte de los requisitos de estudio para obtener el grado de Mestre en Salud Comunitaria, en el Instituto de Salud Colectiva de la Universidad Federal de Bahía, Brasil. También contó con apoyo financiero de una beca de estudio de parte de la Coordinación de Perfeccionamiento de Personal de Nivel Superior (CAPES), del Ministerio de Educación y Cultura de Brasil.

\section{REFERENCIAS}

1. Organización Panamericana de la Salud. La salud en las Américas. Edición de 2002. Washington, DC: OPS 2002. (Publicación científica y técnica 587; 2 vol).

2. World Health Organization. Tuberculosis and sustainable development: the Stop TB initiative. Ginebra, 2000. (Report WHO/CDS/ STB/2000.4).

3. Oliveira HB, Moreira FDC. Recidivas em tuberculose e seus fatores de risco. Rev Panam Salud Publica. 2000;7(4):232-41.
4. Oliveira HB, Moreira FDC. Abandono de tratamento e recidiva da tuberculose: aspectos de episódios prévios, Campinas SP, Brasil, 19931994. Rev Saude Publica. 2000;34(5):437-43.

5. Pulido OF, Peña SR, Rubio RG, González GJ, Pérez-Herrero CJR, Vázquez RJJ, PérezMedel AP. Factores predictivos del abandono del tratamiento antituberculoso en pacientes infectados por el virus de la inmunodeficiencia humana. Rev Clin Esp. 1997;197(3): 163-6.
6. World Health Organization. Global Tuberculose Control: WHO Report 2002. (WHO/ CDS/TB/2002.29).

7. Melo VO, Soares DA, Andrade SM. Avaliação do Programa de Controle da Tuberculose, Londrina, PR, Brasil, 1996. Informe Epidemiológico do SUS Brasil. 1999;8(4): 53-62.

8. Manjarrez EMM, Serrano MV, Cano PG, Verduzco GE, Escandón RC, Escobedo PJ. Principales causas de abandono de tratamiento con- 
tra la tuberculosis pulmonar. Gac Med Mex. 1993;129(1):57-62.

9. Lima MB, Mello DA, Morais APP, Silva WC. Estudo de casos sobre abandono do tratamento da tuberculose: avaliação do atendimento, percepção e conhecimentos sobre a doença na perspectiva dos clientes, Fortaleza, Ceará, Brasil, 1996. Cad Saude Publica. 2001; 17(4):877-85.

10. Natal S, Valente J, Gerhardt G, Penna ML. Modelo de predição para o abandono do tratamento da tuberculose pulmonar. Bol Pneum Sanit. 1999;7(1):67-78.

11. Ferrer $X$, Kirschbaum A, Toro J, Jadue J, Muñoz M, Espinoza A. Adherencia al tratamiento de la tuberculosis del adulto en Santiago, Chile. Bol Oficina Sanit Panam. 1991;111(5):423-31.

12. Deheinzelin D, Takagaki TY, Sartori AMC, Leite OHM, Neto VA, Carvalho CRR. Fatores preditivos de abandono de tratamento por pacientes com tuberculose. Rev Hosp Clin Fac Med Sao Paulo. 1996;51(4):131-135.

13. Nicaragua. Ministerio de Salud, Departamento de Control de Tuberculosis. Manual del Programa de Control de Tuberculosis. Managua; 2001;1-56.

14. Nicaragua. Ministerio de Salud. Informe Anual del Programa de Control de Tuberculosis, $10^{\underline{a}}$ ed. Managua; 2001;1-56.

15. Nicaragua. Ministerio de Salud. Informe Anual del Programa de Control de Tuberculosis, $11^{\mathrm{a}}$ ed. Managua; 2002;1-100.
16. Nicaragua. Ministerio de Salud. Memoria institucional 1997-2001: logros y avances. Managua; 2002.

17. Schlesselman JJ. Case-control studies: design, conduct, analysis. New York: Oxford University Press; 1982.

18. Rothman KJ. Modern epidemiology. Boston: Little-Brown; 1986.

19. Barradas RB. Condições de vida e situação de saúde. Rio de Janeiro: Abrasco; 1997;276.

20. Vitora CG, Huttly SR, Fuchs SC, Olinto MTA. The role of conceptual frameworks in epidemiological analysis: a hierarchical approach. Int J Epidemiol. 1997;26(1):224-27.

21. Burman WJ, Cohn DL, Rietmeijer CA, Judson FN, Sbarbaro JA, Reves RR. Short-term incarceration for the management of noncompliance with tuberculosis treatment. Chest. 1997;112(1):57-62.

22. Ribeiro SA, Amado VM, Camelier AA, Fernandes MMA, Schenkman S. Estudo de casocontrole de indicadores de abandono em doentes com tuberculose. J Pneumologia. 2000;26(6):291-6.

23. Escobar AL, Coimbra CEAJ, Camacho LA, Portela MC. Tuberculose em populações indígenas de Rondônia, Amazônia, Brasil. 1992-1998. Cad Saude Publica. 2001;17(2): 285-98.

24. Rideut M, Menzies R. Factors affecting compliance with preventive treatment for tuberculosis at Mistassini Lake, Quebec, Canada. Clin Invest Med. 1993;17(1):31-6.
25. Burman WJ, Cohn DL, Rietmeiyer CA, Judson FN, Sbarbaro JA, Reves RR. Noncompliance with directly observed therapy for tuberculosis. Chest. 1997;111(5):1168-73.

26. Jiménez GD, López PD, Medrano MJ, Valle MJ. Factores asociados al abandono de pacientes bacilíferos al programa de control de tuberculosis, Managua, Nicaragua, 19981999. Managua: Centro de Investigaciones y Estudios de la Salud (CIES-UNAN); 1992; $1-42$.

27. Albuquerque MFM, Leitão CCS, Campelo ARL, Souza WV, Salustiano A. Fatores prognósticos para o desfecho do tratamento da tuberculose pulmonar em Recife, Pernambuco, Brasil, 1994-1999. Rev Pan Salud Publica. 2001;9(6):368-74.

28. lvarez GGC, Alvarez GJF, Dorantes JJE, Halperin FD. Percepciones y prácticas relacionadas con la tuberculosis y la adherencia al tratamiento en Chiapas México. Salud Publica Mex. 2000;42(6)520-8.

Manuscrito recibido el 23 de agosto de 2004. Aceptado para publicación, tras revisión, el 10 de diciembre de 2004.

ABSTRACT Objective. To identify factors related to dropping out from tuberculosis treatment in health centers in the departments of Managua and Matagalpa, in Nicaragua.

\section{Dropout from tuberculosis treatment in Nicaragua: the results of a comparative study}

\section{Keywords}

Methods. This study matched cases and controls (1:1 ratio) by age and by municipality of treatment. The 251 cases were patients over 15 years of age who had dropped out of tuberculosis treatment, and the 251 controls were patients who had completed pharmacotherapy, during the period of January 1998 to December 2001. Data were obtained on demographic and socioeconomic characteristics, lifestyle habits, and treatment characteristics. The variables were chosen and grouped using a hierarchical theoretical model. Conditional logistic regression analysis was used to calculate odds ratios (ORs) with 95\% confidence intervals (95\% CIs).

Results. The risk factors for dropping out of tuberculosis drug treatment were: male gender (OR, 2.51; 95\% CI, 1.63 to 3.94), an unstable living situation or being homeless (OR, 3.08; 95\% CI, 1.57 to 6.49), changing residence during the treatment period (OR, $4.22 ; 95 \%$ CI, 2.06 to 9.93), consuming alcoholic beverages (OR, 5.25; 95\% CI, 2.43 to 12.94), using illegal drugs (OR, 5.25; 95\% CI, 2.43 to 12.94), difficulty in access to the health services (OR, 2.64; 95\% CI, 1.39 to 5.29), and a negative opinion of the health care received (OR, 5.33; 95\% CI, 1.52 to 28.56 ).

Conclusions. It is essential for the health services in Nicaragua to develop measures to reduce the risk of dropout from treatment. In particular, it is important for the health sector to work with community groups that can support the health sector's activities.

Tuberculosis, drug therapy, patient dropouts, risk factors, Nicaragua. 